\title{
An asymptotically tight bound on the adaptable chromatic number
}

\author{
Michael Molloy* and Giovanna Thron \\ University of Toronto \\ Department of Computer Science \\ 10 King's College Road \\ Toronto, ON, Canada, M5S 3G4 \\ \{molloy, giovanna\}@cs. toronto.edu
}

March 12, 2010

\begin{abstract}
The adaptable chromatic number of a multigraph $G$, denoted $\chi_{a}(G)$, is the smallest integer $k$ such that every edge labeling of $G$ from $[k]=$ $\{1,2, \cdots, k\}$ permits a vertex coloring of $G$ from $[k]$ such that no edge $e=$ $u v$ has $\mathrm{c}(e)=\mathrm{c}(u)=\mathrm{c}(v)$. Hell and Zhu proved that for any multigraph $G$ with maximum degree $\Delta$, the adaptable chromatic number is at most $\lceil\sqrt{e(2 \Delta-1)}\rceil$. We strengthen this to the asymptotically best possible bound of $(1+o(1)) \sqrt{\Delta}$.
\end{abstract}

\section{Introduction}

Hell and Zhu introduced the adaptable chromatic number [15]. Given a (not necessarily proper) edge coloring of a multigraph $G$, we say that a vertex coloring of $G$ is a proper adaptable coloring if there is no edge $e=u v$ in $G$ such that $\mathrm{c}(u)=\mathrm{c}(v)=\mathrm{c}(e)$. The adaptable chromatic number of $G$, denoted $\chi_{a}(G)$, is the minimum number $k$ such that every edge coloring of $G$ using $k$ colors permits an adaptable vertex coloring with the same $k$ colors. Note that $\chi_{a}(G) \leq \chi(G)$, as every proper vertex coloring is a proper adaptable vertex coloring for any edge coloring. Motivations for this problem arise in the contexts of matrix partitions of graphs and full constraint satisfaction problems $[9,10,11]$, job scheduling [16], coloring problems in metric spaces [2], and split colorings [6].

Hell and Zhu prove that $\chi_{a}(G) \leq\lceil\sqrt{e(2 \Delta-1)}\rceil$ where $\Delta$ is the maximum degree of $G$. In this paper we improve this bound to the asymptotically best possible bound of $(1+o(1)) \sqrt{\Delta}$.

*Research supported by an NSERC Discovery Grant. 
We do this by proving a more general result for adaptable list coloring, which is defined naturally. If each vertex $v$ of a multigraph $G$ is given a list of colors, $L(v)$, then a proper adaptable list coloring is a proper adaptable coloring where each vertex's color comes from its list, i.e., $\mathrm{c}(v) \in L(v)$. The adaptable choosability number, denoted $\operatorname{ch}_{a}(G)$, is the smallest integer $k$ such that every edge-coloring and assignment of lists of size $k$ permits a proper adaptable coloring from these lists.

Theorem 1.1. For every $\epsilon>0$, there is a $\Delta_{0}$ such that if $G$ is an edge-colored multigraph of maximum degree $\Delta>\Delta_{0}$ with a list of colors $L(v)$ for each vertex $v \in G$ such that $|L(v)| \geq \sqrt{(1+\epsilon) \Delta}$, then there exists a proper adaptable coloring of $G$ from these lists.

We can see that this bound is asymptotically best possible. For a multigraph example, take the $(k+1)$-clique with $k$ copies of each edge, each colored a different color from $1,2, \ldots, k$. This graph has maximum degree $k^{2}$ and is not $k$-colorable. To see a simple graph example, we refer the reader to [14], where the authors prove that $\chi_{a}\left(K_{n}^{2}\right)=n$ if there is a projective plane of order $n$ or $n+1$. Note that the maximum degree of $K_{n}^{2}$ is $(n-1)^{2}$.

An analogous problem for list coloring was introduced by Reed [21], where he proved that for any $d$, if a graph $G$ has lists $L(v)$ for each vertex $v$ such that (i) for each color $c \in L(v)$, the number of neighbors $u$ of $v$ such that $c \in L(u)$ is at most $d$, and (ii) $|L(v)| \geq 2 e d$, then $G$ can be colored. Haxell [13] improved this result by showing $G$ can be colored if $|L(v)|=2 d$. Reed and Sudakov [22] later showed that for any $\epsilon>0$, if $d$ is sufficiently large and $|L(v)|=(1+\epsilon) d$, then $G$ can be colored.

The result of [22] is a special case of Theorem 1.1, since we can convert an instance of the list coloring problem, $G$, to an instance of the adaptable list coloring problem, $G^{\prime}$. Let $G^{\prime}$ have the same vertex set as $G$ and give each vertex the same list that it had in $G$. For each edge $u v$ in $G$ and each color $c \in L(u) \cap L(v)$, let $G^{\prime}$ have a copy of the edge $u v$ and give it the color $c$. Now $G^{\prime}$ has maximum degree at most $\Delta \leq d l$, where $l$ is the maximum size of the lists, and can be adaptively colored if and only if $G$ has a coloring from the given lists. Note that $l \geq \sqrt{(1+\epsilon) \Delta}$ is equivalent to $l \geq(1+\epsilon) d$.

Our proof of Theorem 1.1 follows the approach taken in [22].

Lower bounds for the adaptable chromatic number for the general multigraph have also been studied; the order of $\chi_{a}(G)$ is at least $\chi(G) / \sqrt{n \log \chi(G)}[12]$. A lot of attention has been given to the study of complete graphs $[2,6,15]$; the best upper and lower bounds are both of order $\sqrt{n}[6]$. Planar graphs were first looked at in [15], and later [8] proved that 4 colors were sufficient without using the Four Color Theorem. The adaptable chromatic number of a graph can be arbitrarily high for any girth [15], and can be as large as the chromatic number [14]. In $[15,16]$, graphs with $\chi_{a}(G)=2$ and graphs with $\operatorname{ch}_{a}(G)=2$ were characterized; recognizing graphs with $\chi_{a}(G)=t$ for $t>2$ is NP-complete [15].

Remark: Our proof makes use of the Lovász Local Lemma. It is straightforward to apply the results of $[19,20]$ to obtain a randomized algorithm for 
finding an adaptable coloring of a graph which satisfies the conditions of Theorem 1.1; the expected running time is $O\left(|V| \Delta^{2} \log ^{2} \Delta+|E|\right)$. We can similarly apply the results of [4] to get a polytime deterministic algorithm.

\section{Probabilistic Tools}

In this section, we describe some important probabilistic tools that will be used extensively in the proof of Theorem 1.1. One of the most important probabilistic tools is the Lovász Local Lemma, which was first proved in [7] and is presented in many places including $[1,18]$.

Lovász Local Lemma. Let $A_{1}, A_{2}, \ldots, A_{n}$ be a set of "bad" events in a probability space. Suppose that each event $A_{i}$ is mutually independent of all the other events $A_{j}$ but at most $d$, and that $\operatorname{Pr}\left(A_{i}\right) \leq p$ for all $1 \leq i \leq n$. If ep $(d+1) \leq 1$, then with positive probability, no event $A_{i}$ holds.

We use two important concentration bounds. The first is the commonly used Chernoff Bound [5]. Instead of using the original statement we use the (weaker) version found in [18]. Here, $B I N(n, p)$ is the sum of $n$ independent variables, each equal to 1 with probability $p$ and 0 otherwise.

Chernoff Bound. For any $0 \leq t \leq n p$ :

$$
\operatorname{Pr}(|B I N(n, p)-n p|>t)<2 e^{-\frac{t^{2}}{3 n p}}
$$

Our next tool is Talagrand's Inequality [23]. Instead of using the original statement we use the (weaker) version found in [17] (see Equation (2) of Section $3.1)$.

Talagrand's Inequality. Let $X$ be a non-negative random variable determined by the independent trials $T_{1}, T_{2}, \ldots, T_{n}$. Suppose that for every set of possible outcomes of the trials, we have:

1. changing the outcome of any one trial can affect $X$ by at most c; and

2. for each $s>0$, if $X \geq s$, then there is a set of at most rs trials whose outcomes certify that $X \geq s$.

Then for any $t \geq 0$ where $\frac{t}{2} \geq 20 c+\sqrt{r E(X)}+64 c^{2} r$, we have

$$
\operatorname{Pr}(|X-E(X)|>t) \leq 4 e^{-\frac{t^{2}}{32 c^{2} r(E(X)+t)}} .
$$

\section{A Simpler Bound}

Reed's initial result [21] can be generalized to the following lemma. Note that while this result is weaker than Theorem 1.1 above, this result holds for all $\Delta$ rather than just sufficiently large $\Delta$. This theorem is also an essential part of proving Theorem 1.1. 
Lemma 3.1. If $G$ is an edge-colored multigraph of maximum degree $\Delta>0$ with a list of colors $L(v)$ for each vertex $v$ in $G$ such that $|L(v)| \geq \sqrt{e(2 \Delta-1)}$, then there exists a proper adaptable coloring of $G$ from these lists.

The proof of this theorem is nearly identical to that of the analogous theorem for regular coloring which can be seen in [21]. In [15, 2], the authors prove the result for regular adaptable coloring; the proof is the same.

Proof. This is a straightforward application of the Lovász Local Lemma. Let $G$ be an edge-colored graph with lists for each vertex satisfying the conditions of the theorem. Truncate the lists to size $l=\lceil\sqrt{2 e(\Delta-1)}\rceil$. Assign each vertex $v$ in $G$ a color uniformly at random from $\{1,2, \ldots, l\}$.

For each edge $e=u v$, let $A_{e}$ be the bad event where $\mathrm{c}(u v)=\mathrm{c}(u)=\mathrm{c}(v)$. Let $\mathcal{E}$ be the set of all such events. We must determine the probabilities and dependencies of each event $A_{e}$ corresponding to the edge $e=u v$. Let $c=\mathrm{c}(e)$. If $c \notin L_{u}$ or $c \notin L_{v}$, then $\operatorname{Pr}\left(A_{e}\right)=0$, and this is dependent on nothing. If $c \in L_{u}$ and $c \in L_{v}$, then $\operatorname{Pr}\left(A_{e}\right)=\frac{1}{l^{2}}$. To see the dependency of $A_{e}$, define the following:

$$
\begin{aligned}
& D_{u}=\left\{A_{f}: f=u u^{\prime} \neq e \text { is an edge in } G \text { and } \mathrm{c}(f) \in L_{u} \cap L_{u^{\prime}}\right\} \\
& D_{v}=\left\{A_{f}: f=v v^{\prime} \neq e \text { is an edge in } G \text { and } \mathrm{c}(f) \in L_{v} \cap L_{v^{\prime}}\right\}
\end{aligned}
$$

$A_{e}$ is mutually independent of all events in $\mathcal{E}-\left|D_{u}\right|-\left|D_{v}\right|$. Note that $\left|D_{u}\right| \leq$ $\Delta-1$ and $\left|D_{v}\right| \leq \Delta-1$. So $A_{e}$ is dependent on at most $2 \Delta-2$ events.

If $p$ is the probability of each bad event occurring and $d$ is the number of events each bad event is dependent on, we get

$$
e p(d+1) \leq e\left(\frac{1}{l^{2}}\right)(2 \Delta-1) \leq e\left(\frac{1}{e(2 \Delta-1)}\right)(2 \Delta-1)=1,
$$

so we can apply the Lovász Local Lemma.

\section{Proof of Main Theorem}

Instead of specifying $\Delta_{0}$, we, several times throughout the proof, use the fact that $\Delta$ is a sufficiently large constant.

To prove Theorem 1.1, we use a random coloring approach like the one used in [22] and many other places (see e.g. [18]). We iteratively color a small portion of the vertices, uncoloring any vertices which create collisions. For each vertex $v$, we maintain a list $L_{i}(v)$ of available colors at iteration $i$, each of which have size $L_{i}$ which is specified below.

Wasteful Coloring Procedure (ith iteration).

1. For each uncolored vertex $v$, activate $v$ with probability $\frac{1}{\ln \Delta}$.

2. For each activated vertex $v$, assign $v$ a color uniformly at random from $L_{i}(v)$. 
3. For each activated vertex $v$ : If $v$ is assigned the color $c$, for each uncolored neighbor $u$ of $v$ such that $\mathrm{c}(u v)=c$, remove $c$ from $L(u)$.

4. Uncolor every vertex $v$ which receives the same color $c$ as a neighbor $u$ where $\mathrm{c}(u v)=c$.

5. Truncate the lists so they are all of size $L_{i}$. We do this in a very specific way, by removing the colors $c$ for which the parameter $t_{i}(v, c)$ (defined below) is greatest.

Note that this procedure is wasteful since we remove $v$ 's color from all of $v$ 's neighbors' lists in Step 3 even if we uncolor $v$ in Step 4. We could keep these colors, as they are still usable, but removing them makes the calculations simpler. Below we will prove that with high probability after Step 4 every list has size at least $L_{i}$ and thus Step 5 is possible.

Now we define several useful parameters for each vertex $v$ and color $c$. All parameters refer to the value at the end of iteration $i$.

$$
\begin{aligned}
L_{i}(v) & =v \text { 's list of colors } \\
N_{i}(v, c) & =\left\{\text { uncolored neighbors } u \text { of } v \text { such that } c=\mathrm{c}(u v) \in L_{i}(u)\right\} \\
t_{i}(v, c) & =\left|N_{i}(v, c)\right| \\
x_{i}(v) & =\# \text { of uncolored neighbors } u \text { of } v \text { such that } \mathrm{c}(u v) \in L_{i}(u) \cap L_{i}(v) \\
& =\sum_{c \in L_{i}(v)} t_{i}(v, c)
\end{aligned}
$$

Note that initially we have $x_{0}(v) \leq \Delta$ and $l_{0}(v) \geq \sqrt{(1+\epsilon) \Delta}$ for each vertex $v$.

Proving the concentration of $x_{i}$ is made more complicated if the value of a $t_{i}$ is too large, as will be seen in the proof of lemma 5.3. One trial can affect $x_{i}$ by as much as the largest $t_{i}$, and we need this value to not be too large. The following proposition demonstrates that we will be able to modify the lists so that there are no large $t_{i}$ 's. Since the $t_{i}$ 's only decrease with each iteration, we only have to do this once at the beginning. We choose $\Delta^{3 / 5}$ as our threshold for convenience, but $\Delta$ to any power strictly between $\frac{1}{2}$ and 1 would suffice.

Proposition 4.1. For each vertex $v$, the number of colors $c$ for which $t_{0}(v, c)<$ $\Delta^{3 / 5}$ is at least $\sqrt{\left(1+\frac{\epsilon}{2}\right) \Delta}$.

Proof. Note that for each vertex $v$ there are at most $\Delta^{2 / 5} c^{\prime}$ s such that $t_{0}(v, c) \geq$ $\Delta^{3 / 5}$. So the number of $c$ 's where $t_{0}(v, c)<\Delta^{3 / 5}$ is at least $\sqrt{(1+\epsilon) \Delta}-\Delta^{2 / 5} \geq$ $\sqrt{\left(1+\frac{\epsilon}{2}\right) \Delta}$ for sufficiently large $\Delta$.

Proposition 4.1 permits the following modification to our graph $G$.

Modification 1. Remove from each list $L(v)$ any color $c$ for which $t_{0}(v, c) \geq \Delta^{3 / 5}$. Arbitrarily truncate all the lists to have exactly $\left\lceil\sqrt{\left(1+\frac{\epsilon}{2}\right) \Delta}\right\rceil$ elements. 
Because of this truncation, it will be convenient to do the following reparameterization:

\section{Change of Variables: $\alpha=\frac{\epsilon}{2}$.}

The reader may notice that small $t_{i}$ 's also pose a problem. They will be dealt with in Section 5 by the introduction of $L_{i}^{\prime}(v)$.

$L_{i}$ will be the size of all the lists at the end of iteration $i$, and $X_{i}$ will be an upper bound for $x_{i}(v)$. They are defined by the following recurrences.

$$
\begin{aligned}
L_{0} & =[\sqrt{(1+\alpha) \Delta}] \\
X_{0} & =\Delta \\
L_{i+1} & =\left(1-\frac{1}{\left(1+\frac{3 \alpha}{4}\right) \ln \Delta}\right) L_{i} \\
X_{i+1} & =\left(1-\frac{1}{\left(1+\frac{\alpha}{2}\right) \ln \Delta}\right)^{2} X_{i}
\end{aligned}
$$

We now introduce a new parameter.

$$
l_{i}(v)=\text { size of } v \text { 's list right before step } 5 \text { in iteration } i
$$

We would like to maintain the following property.

Property $P(i)$. For each uncolored vertex $v$ of the graph $G$, the following is true:

$$
l_{i}(v) \geq L_{i} \text { and } x_{i}(v) \leq X_{i}
$$

Lemma 4.2. With positive probability, at the end of each iteration $i$ for $0 \leq$ $i \leq\left\lceil\frac{10}{\alpha} \ln \Delta\right\rceil$, Property $P(i)$ holds.

We now prove the main theorem using this lemma.

Proof of Theorem 1.1. Carry out the wasteful coloring procedure up through iteration $i^{\prime}=\left[\frac{10}{\alpha} \ln \Delta\right]$. Property $P(i)$ ensures that we can carry out Step 5 . At the end of iteration $i^{\prime}$, each vertex $v$ has $\left|L_{i^{\prime}}(v)\right|=L_{i^{\prime}}$ and $x_{i^{\prime}}(v) \leq X_{i^{\prime}}$. We will show that the ratio of $x_{i^{\prime}}(v)$ to $\left|L_{i^{\prime}}(v)\right|^{2}$ is at most $\frac{1}{2 e}$; thus we can complete the coloring using Lemma 3.1.

$$
\begin{aligned}
\frac{x_{i^{\prime}}(v)}{\left|L_{i^{\prime}}(v)\right|^{2}} \leq \frac{X_{i^{\prime}}}{L_{i^{\prime}}^{2}} & =\frac{\left(1-\frac{1}{\left(1+\frac{\alpha}{2}\right) \ln \Delta}\right)^{2 i^{\prime}} X_{0}}{\left(1-\frac{1}{\left(1+\frac{3 \alpha}{4}\right) \ln \Delta}\right)^{2 i^{\prime}} L_{0}^{2}} \\
& =\left(\frac{1-\frac{1}{\left(1+\frac{\alpha}{2}\right) \ln \Delta}}{1-\frac{1}{\left(1+\frac{3 \alpha}{4}\right) \ln \Delta}}\right)^{2 i^{\prime}} \cdot \frac{\Delta}{(1+\alpha) \Delta} \\
& <\left(\frac{\left(1+\frac{\alpha}{2}\right)\left(1+\frac{3 \alpha}{4}\right) \ln \Delta-\left(1+\frac{3 \alpha}{4}\right)}{\left(1+\frac{\alpha}{2}\right)\left(1+\frac{3 \alpha}{4}\right) \ln \Delta-\left(1+\frac{\alpha}{2}\right)}\right)^{2 i^{\prime}}
\end{aligned}
$$




$$
\begin{aligned}
& =\left(1-\frac{\frac{\alpha}{4}}{\left(1+\frac{\alpha}{2}\right)\left(1+\frac{3 \alpha}{4}\right) \ln \Delta-\left(1+\frac{\alpha}{2}\right)}\right)^{2 i^{\prime}} \\
& \leq\left(1-\frac{\alpha}{10 \ln \Delta}\right)^{2 \cdot \frac{10}{\alpha} \ln \Delta} \\
& \leq e^{-2}<\frac{1}{2 e}
\end{aligned}
$$

To prove Lemma 4.2 , we need to show that with high probability $x_{i}$ decreases quickly enough while $l_{i}$ does not decrease too quickly. The concentration lemmas, which we prove in Section 5, are as follows.

Lemma 4.3. For each vertex $v$ and $0 \leq i \leq\left\lceil\frac{10}{\alpha} \ln \Delta\right\rceil$, if $P(i)$ holds, then

$$
\operatorname{Pr}\left(l_{i+1}(v)<L_{i+1}\right)<\Delta^{-\ln \Delta}
$$

Lemma 4.4. For each vertex $v$ and $0 \leq i \leq\left\lceil\frac{10}{\alpha} \ln \Delta\right\rceil$, if $P(i)$ holds, then

$$
\operatorname{Pr}\left(x_{i+1}(v)>X_{i+1}\right)<\Delta^{-\ln \Delta}
$$

The proof of Lemma 4.2 follows easily from Lemmas 4.3 and 4.4.

Proof of Lemma 4.2. We prove this by induction on $i$. The base case $i=0$ is trivially true. Let $A_{v}$ be the bad event that $l_{i+1}(v)<L_{i+1}$ and let $B_{v}$ be the bad event that $x_{i+1}(v)>X_{i+1}$. By Lemmas 4.3 and 4.4 the probability of each of these bad events is at most $\Delta^{-\ln \Delta}$. $A_{v}$ and $B_{v}$ are easily seen to be mutually independent from all $A_{u}$ and $B_{v}$ where $u$ is a distance greater than 4 from $v$. Since the maximum degree of $G$ is at most $\sqrt{(1+\epsilon) \Delta} \cdot \Delta<\Delta^{2}, A_{v}$ and $B_{u}$ are mutually independent from all but at most $\Delta^{8}$ events. So, as

$$
e \cdot \Delta^{-\ln \Delta} \cdot \Delta^{8}<1 \Delta \text { for sufficiently large, }
$$

we can apply the Lovász Local Lemma.

\section{Proofs of the Concentration Lemmas}

The bulk of the proof is in the concentration lemmas.

\section{$5.1 \quad$ Helpful Facts}

We start with three facts that are used several times throughout the proofs of our concentration lemmas.

Since $L_{0}=\sqrt{(1+\alpha) \Delta}$ and

$$
L_{i}=\left(1-\frac{1}{\left(1+\frac{3 \alpha}{4}\right) \ln \Delta}\right)^{i} \cdot L_{0}
$$




$$
\geq\left(1-\frac{1}{\left(1+\frac{3 \alpha}{4}\right) \ln \Delta}\right)^{\frac{10}{\alpha} \ln \Delta+1} \cdot \sqrt{\Delta}=\Theta(\sqrt{\Delta}),
$$

we get the following.

Fact 1. $L_{i}=\Theta(\sqrt{\Delta})$ for all $i$ such that $0 \leq i \leq\left\lceil\frac{10}{\alpha} \ln \Delta\right\rceil$.

Similarly, as $X_{0}=\Delta$ and

$$
\begin{aligned}
X_{i} & =\left(1-\frac{1}{\left(1+\frac{\alpha}{2}\right) \ln \Delta}\right)^{2 i} \cdot X_{0} \\
& \geq\left(1-\frac{1}{\left(1+\frac{\alpha}{2}\right) \ln \Delta}\right)^{\frac{20}{\alpha} \ln \Delta+1} \cdot \Delta=\Theta(\Delta),
\end{aligned}
$$

we get a similar fact for $X_{i}$.

Fact 2. $X_{i}=\Theta(\Delta)$ for all $i$ such that $0 \leq i \leq\left\lceil\frac{10}{\alpha} \ln \Delta\right\rceil$.

Finally, the ratio of $X_{i}$ to $L_{i}^{2}$ comes up several times and we use the following.

$$
\frac{X_{i}}{L_{i}^{2}}=\frac{\left(1-\frac{1}{\left(1+\frac{\alpha}{2}\right) \ln \Delta}\right)^{2 i^{\prime}} X_{0}}{\left(1-\frac{1}{\left(1+\frac{3 \alpha}{4}\right) \ln \Delta}\right)^{2 i^{\prime}} L_{0}^{2}} \leq \frac{X_{0}}{L_{0}^{2}}=\frac{1}{1+\alpha}
$$

This yields our final useful fact.

Fact 3. $\frac{X_{i}}{L_{i}^{2}} \leq \frac{1}{1+\alpha}$ for all $i$ such that $0 \leq i \leq\left\lceil\frac{10}{\alpha} \ln \Delta\right\rceil$.

\subsection{Proof of Lemma 4.3}

We start with bounding the expected value of $l_{i+1}(v)$.

Lemma 5.1. For each vertex $v$ and $0 \leq i \leq\left\lceil\frac{10}{\alpha} \ln \Delta\right\rceil$, if $P(i)$ holds, then,

$$
E\left(l_{i+1}(v)\right) \geq\left(1-\frac{1}{(1+\alpha) \ln \Delta}\right) L_{i} .
$$

Proof.

$$
\begin{aligned}
E\left(l_{i+1}(v)\right) & =\sum_{c \in L_{i}(v)} \operatorname{Pr}(\text { no neighbor } u \text { of } v \text { is assigned color } c) \\
& =\sum_{c \in L_{i}(v)} \prod_{u \in N_{i}(v, c)}(1-\operatorname{Pr}(u \text { is activated and assigned } c)) \\
& =\sum_{c \in L_{i}(v)} \prod_{u \in N_{i}(v, c)}\left(1-\frac{1}{\ln \Delta \cdot L_{i}}\right)
\end{aligned}
$$




$$
=\sum_{c \in L_{i}(v)}\left(1-\frac{1}{\ln \Delta \cdot L_{i}}\right)^{t_{i}(v, c)}
$$

In the following claim, we show that we can use $\frac{x_{i}(v)}{L_{i}}$ in place of $t_{i}(v, c)$. Note that this quantity need not be an integer.

Claim 5.1.1. Given $x_{i}(v)=\sum_{c \in L_{i}(v)} t_{i}(v, c)$, the above bound is minimized over the rationals when all the $t_{i}$ 's are equal.

Proof of claim. Let $c_{1}$ and $c_{2}$ be two colors in $L_{i}(v)$ such that the following is true.

$$
t_{i}\left(v, c_{1}\right)>\frac{x_{i}(v)}{L_{i}} \text { and } t_{i}\left(v, c_{2}\right)<\frac{x_{i}(v)}{L_{i}}
$$

Let $t_{1}=t_{i}\left(v, c_{1}\right), t_{2}=t_{i}\left(v, c_{2}\right)$ and $\gamma=1-\frac{1}{\ln \Delta \cdot L_{i}}$. Define $t_{i}^{\prime}$ as follows, where $\beta=\min \left(t_{1}-\frac{x_{i}(v)}{L_{i}}, \frac{x_{i}(v)}{L_{i}}-t_{2}\right)$.

$$
t_{i}^{\prime}(v, c)= \begin{cases}t_{1}-\beta, & \text { if } c=c_{1} \\ t_{2}+\beta, & \text { if } c=c_{2} \\ t_{i}(v, c), & \text { o.w. }\end{cases}
$$

Let $D=\sum_{c \in L_{i}(v)} \gamma^{t_{i}(v, c)}-\sum_{c \in L_{i}(v)} \gamma^{t_{i}^{\prime}(v, c)}$. It suffices to show that $D \geq 0$.

$$
\begin{aligned}
D & =\gamma^{t_{1}}+\gamma^{t_{2}}-\gamma^{t_{1}-\beta}-\gamma^{t_{2}+\beta} \\
& =-\gamma^{t_{1}-\beta}\left(1-\gamma^{\beta}\right)+\gamma^{t_{2}}\left(1-\gamma^{\beta}\right) \\
& =\left(1-\gamma^{\beta}\right)\left(\gamma^{t_{2}}-\gamma^{t_{1}-\beta}\right)
\end{aligned}
$$

Since $t_{1}-\beta \geq t_{2}$ and $0<\gamma<1, D \geq 0$.

Using Claim 5.1.1, we can continue our computation of the bound on the expected value of $l_{i+1}(v)$.

$$
\begin{aligned}
E\left(l_{i+1}(v)\right) & \geq \sum_{c \in L_{i}(v)}\left(1-\frac{1}{\ln \Delta \cdot L_{i}}\right)^{\frac{x_{i}(v)}{L_{i}}} \\
& \left.\geq \sum_{c \in L_{i}(v)}\left(1-\frac{1}{\ln \Delta \cdot L_{i}}\right)^{\frac{X_{i}}{L_{i}}} \text { [by Property } P(i)\right] \\
& =L_{i}\left(1-\frac{1}{\ln \Delta \cdot L_{i}}\right)^{\frac{X_{i}}{L_{i}}} \\
& \left.\geq L_{i}\left(1-\frac{X_{i}}{\ln \Delta \cdot L_{i}^{2}}\right) \text { for } \Delta \text { sufficiently large [by Facts } 1 \& 2\right] \\
& \geq L_{i}\left(1-\frac{1}{(1+\alpha) \ln \Delta}\right)[\text { by Fact } 3]
\end{aligned}
$$

And this completes our proof of Lemma 5.1. 
We now show that the lists stay large enough by proving Lemma 4.3.

Proof of Lemma 4.3. Instead of showing the concentration of $l_{i+1}$ directly, it is more convenient to work with $R=L_{i}-l_{i+1}(v)$, the number of colors which leave $v$ 's list. By the linearity of expectation and Lemma 5.1, we have $E(R)=$ $L_{i}-E\left(l_{i+1}(v)\right) \leq L_{i} \cdot\left(\frac{1}{(1+\alpha) \ln \Delta}\right)<\frac{L_{i}}{\ln \Delta}$.

Changing the assignment of any vertex can affect $R$ by at most 1 , as changing the assignment of one of $v$ 's neighbors can at most change $R$ by 1 , and changing any other vertex has no effect. Furthermore, for any $s$, to certify $R \geq s$, we can use $s$ neighbors of $v$ which were assigned different colors. So Talagrand's Inequality with $c=r=1$ and $t=\frac{L_{i}}{\ln ^{2} \Delta}$ yields:

$$
\begin{aligned}
\operatorname{Pr}\left(|R-E(R)|>\frac{L_{i}}{\ln ^{2} \Delta}\right) & <4 e^{-\frac{L_{i}^{2} / \ln ^{4} \Delta}{32\left(L_{i} / \ln \Delta+L_{i} / \ln ^{2} \Delta\right)}} \\
& <4 e^{-\frac{L_{i}}{64 \ln ^{3} \Delta}} \\
& \left.<e^{-\ln ^{2} \Delta}=\Delta^{-\ln \Delta} \text { [by Fact } 1\right]
\end{aligned}
$$

Since $R=L_{i}-l_{i+1}(v)$ and $E(R)=L_{i}-E\left(l_{i+1}(v)\right)$, Lemma 5.1 yields:

$$
\begin{aligned}
& \operatorname{Pr}\left(l_{i+1}(v)<\left(1-\frac{1}{\left(1+\frac{3 \alpha}{4}\right) \ln \Delta}\right) L_{i}\right) \\
& \leq \operatorname{Pr}\left(l_{i+1}(v)<\left(1-\frac{1}{(1+\alpha) \ln \Delta}\right) L_{i}-\frac{L_{i}}{\ln ^{2} \Delta}\right) \\
& \leq \operatorname{Pr}\left(l_{i+1}(v)<E\left(l_{i+1}(v)\right)-\frac{L_{i}}{\ln ^{2} \Delta}\right) \\
& \leq \operatorname{Pr}\left(\left|l_{i+1}(v)-E\left(l_{i+1}(v)\right)\right|>\frac{L_{i}}{\ln ^{2} \Delta}\right) \\
& =\operatorname{Pr}\left(|R-E(R)|>\frac{L_{i}}{\ln ^{2} \Delta}\right) \\
& <\Delta^{-\ln \Delta}
\end{aligned}
$$

\subsection{Proof of Lemma 4.4}

The concentration of $x_{i}$ is more involved. It is convenient to split the analysis into steps by introducing the following parameters.

$$
\begin{aligned}
L_{i}^{*}(v) & =v \text { 's list of colors right before Step } 5 \\
y_{i}(v) & =\sum_{c \in L_{i}^{*}(v)} t_{i-1}(v, c)
\end{aligned}
$$

The parameter $y_{i}$ is like $x_{i}$, except we are just reducing the size of the list, and not decreasing the $t_{i}$ 's, and we are looking at the value before the truncation 
step. This allows us to analyze the rate of decrease of the lists and the $t_{i}$ 's separately. To show that $x_{i}$ decreases enough, we show that both $y_{i}$ and $t_{i}$ decrease enough. We will then factor in the effect of the truncation step. We start our analysis by computing a bound on the expected value of $y_{i+1}$.

Lemma 5.2. For each vertex $v$ and $0 \leq i \leq\left\lceil\frac{10}{\alpha} \ln \Delta\right\rceil$, if $P(i)$ holds, then

$$
E\left(y_{i+1}(v)\right) \leq\left(\frac{E\left(l_{i+1}(v)\right)}{L_{i}}+\frac{1}{\ln ^{3} \Delta}\right) X_{i}
$$

Proof. Bound the expected value of $y_{i+1}(v)$ with the following calculations. Remember that a color $c$ is removed from $v$ 's list if a neighbor is colored $c$.

$$
\begin{aligned}
E\left(y_{i+1}(v)\right) & =\sum_{c \in L_{i}(v)} \operatorname{Pr}\left(c \in L_{i+1}(v)\right) \cdot t_{i}(v, c) \\
& \leq \sum_{c \in L_{i}(v)}\left[\prod_{u \in N_{i}(v, c)}\left(1-\frac{1}{\ln \Delta \cdot L_{i}}\right)\right] \cdot t_{i}(v, c) \\
& =\sum_{c \in L_{i}(v)}\left(1-\frac{1}{\ln \Delta \cdot L_{i}}\right)^{t_{i}(v, c)} \cdot t_{i}(v, c)
\end{aligned}
$$

We show that this bound on $E\left(y_{i+1}(v)\right)$ is maximized over integers when

$$
t_{i}(v, c)=\left\lceil\frac{x_{i}(v)}{L_{i}}\right\rceil \text { or }\left\lfloor\frac{x_{i}(v)}{L_{i}}\right\rfloor
$$

for all vertices $v$. Suppose, to the contrary, that there are two colors $c_{1}, c_{2} \in$ $L_{i}(v)$ such that

$$
t_{i}\left(v, c_{1}\right)>t_{i}\left(v, c_{2}\right)+1 .
$$

Let $t_{1}=t_{i}\left(v, c_{1}\right)$ and $t_{2}=t_{i}\left(v, c_{2}\right)$. Define $t_{i}^{\prime}$ as follows.

$$
t_{i}^{\prime}(v, c)= \begin{cases}t_{1}-1, & \text { if } c=c_{1} \\ t_{2}+1, & \text { if } c=c_{2} \\ t_{i}(v, c), & \text { o.w. }\end{cases}
$$

Let

$$
D=\sum_{c \in L_{i}(v)}\left(1-\frac{1}{\ln \Delta \cdot L_{i}}\right)^{t_{i}^{\prime}(v, c)} t_{i}^{\prime}(v, c)-\sum_{c \in L_{i}(v)}\left(1-\frac{1}{\ln \Delta \cdot L_{i}}\right)^{t_{i}(v, c)} t_{i}(v, c) .
$$

It suffices to show that $D \geq 0$.

$$
\begin{aligned}
D= & \left(1-\frac{1}{\ln \Delta \cdot L_{i}}\right)^{t_{1}-1}\left(t_{1}-1\right)+\left(1-\frac{1}{\ln \Delta \cdot L_{i}}\right)^{t_{2}+1}\left(t_{2}+1\right) \\
& -\left(1-\frac{1}{\ln \Delta \cdot L_{i}}\right)^{t_{1}} t_{1}-\left(1-\frac{1}{\ln \Delta \cdot L_{i}}\right)^{t_{2}} t_{2}
\end{aligned}
$$




$$
\begin{aligned}
= & \left(1-\frac{1}{\ln \Delta \cdot L_{i}}\right)^{t_{1}-1}\left[t_{1}-1-\left(1-\frac{1}{\ln \Delta \cdot L_{i}}\right) t_{1}\right] \\
& +\left(1-\frac{1}{\ln \Delta \cdot L_{i}}\right)^{t_{2}}\left[\left(1-\frac{1}{\ln \Delta \cdot L_{i}}\right)\left(t_{2}+1\right)-t_{2}\right] \\
= & \left(1-\frac{1}{\ln \Delta \cdot L_{i}}\right)^{t_{1}-1}\left(\frac{t_{1}}{\ln \Delta \cdot L_{i}}-1\right)+\left(1-\frac{1}{\ln \Delta \cdot L_{i}}\right)^{t_{2}}\left(1-\frac{t_{2}+1}{\ln \Delta \cdot L_{i}}\right)
\end{aligned}
$$

Property $P(i)$ and Fact 3 yields that the second term here is positive by the following argument.

$$
\frac{t_{2}+1}{\ln \Delta \cdot L_{i}} \leq \frac{\frac{x_{i}(v)}{L_{i}}}{\ln \Delta \cdot L_{i}} \leq \frac{X_{i}}{L_{i}^{2} \ln \Delta} \leq \frac{1}{(1+\alpha) \ln \Delta}<1
$$

for sufficiently large $\Delta$.

Thus if $D<0$ then the first term is negative. So assume $\frac{t_{1}}{\ln \Delta \cdot L_{i}}-1<0$. Note that by our definitions of $t_{1}$ and $t_{2}$ we have that $t_{1}-1 \geq t_{2}$. So we get the following.

$$
\begin{aligned}
D & \geq\left(1-\frac{1}{\ln \Delta \cdot L_{i}}\right)^{t_{2}}\left(\frac{t_{1}}{\ln \Delta \cdot L_{i}}-1\right)+\left(1-\frac{1}{\ln \Delta \cdot L_{i}}\right)^{t_{2}}\left(1-\frac{t_{2}+1}{\ln \Delta \cdot L_{i}}\right) \\
& =\left(1-\frac{1}{\ln \Delta \cdot L_{i}}\right)^{t_{2}}\left[\left(\frac{t_{1}}{\ln \Delta \cdot L_{i}}-1\right)+\left(1-\frac{t_{2}+1}{\ln \Delta \cdot L_{i}}\right)\right] \\
& =\left(1-\frac{1}{\ln \Delta \cdot L_{i}}\right)^{t_{2}}\left(\frac{\left.t_{1}-1-t_{2}\right)}{\ln \Delta \cdot L_{i}}\right)>0
\end{aligned}
$$

So the bound on $E\left(y_{i+1}(v)\right)$ is maximized over the integers when all $t_{i}$ 's are within one of each other; i.e., $t_{i}(v, c)=\left\lceil x_{i}(v) / L_{i}\right\rceil$ or $\left\lfloor x_{i}(v) / L_{i}\right\rfloor$ for all vertices $v$. Using Equation 1 from the proof of Lemma 5.1 as a bound on $E\left(l_{i+1}(v)\right)$, we continue computing the bound on $E\left(y_{i+1}(v)\right)$ as follows.

$$
\begin{aligned}
E\left(y_{i+1}(v)\right) & \leq \sum_{c \in L_{i}(v)}\left(1-\frac{1}{\ln \Delta \cdot L_{i}}\right)^{\left.\frac{x_{i}(v)}{L_{i}}\right\rfloor}\left\lceil\frac{x_{i}(v)}{L_{i}}\right\rceil \\
& \leq \sum_{c \in L_{i}(v)}\left(1-\frac{1}{\ln \Delta \cdot L_{i}}\right)^{\frac{x_{i}(v)}{L_{i}}-1}\left(\frac{x_{i}(v)}{L_{i}}+1\right) \\
& \leq \frac{E\left(l_{i+1}(v)\right)}{\left(1-\frac{1}{\ln \Delta \cdot L_{i}}\right)}\left(\frac{X_{i}}{L_{i}}+1\right)[\text { by the above and } P(i)] \\
& \leq\left(\frac{E\left(l_{i+1}(v)\right)}{L_{i}}+\frac{1}{\ln ^{3} \Delta}\right) X_{i}[\text { by Facts } 1 \text { and } 2] .
\end{aligned}
$$

As mentioned above, proving that with high probability $y_{i}$ and the $t_{i}$ 's decrease enough (Theorems 5.3 and 5.4 below) is simplified if we disregard small $t_{i}$ 's. We introduce the following parameters:

$$
L_{i}^{\prime}(v)=\left\{c \in L_{i}(v): t_{i}(v, c) \geq \Delta^{2 / 5}\right\}
$$




$$
\begin{aligned}
x_{i}^{\prime}(v) & =\sum_{c \in L_{i}^{\prime}(v)} t_{i}(v, c) \\
L_{i}^{* \prime}(v) & =\left\{c \in L_{i}^{*}(v): t_{i-1}(v, c) \geq \Delta^{2 / 5}\right\} \\
y_{i}^{\prime}(v) & =\sum_{c \in L_{i}^{* \prime}(v)} t_{i-1}(v, c)
\end{aligned}
$$

The parameters $x_{i}^{\prime}$ and $y_{i}^{\prime}$ are the same as $x_{i}$ and $y_{i}$, respectively, except we have disregarded the small $t_{i}$ 's. Note that $x_{i}^{\prime}$, like $x_{i}$, is measured after Step 5. On the other hand, $y_{i}^{\prime}$, like $y_{i}$, is measured before Step 5 . The choice of $\Delta^{2 / 5}$ is used for convenience, but anything between $\ln ^{3} \Delta$ and $o(\sqrt{\Delta})$ would work.

Note that $E\left(y_{i+1}^{\prime}(v)\right) \leq E\left(y_{i+1}(v)\right)$, so Lemma 5.1 yields:

$$
E\left(y_{i+1}^{\prime}(v)\right) \leq\left(\frac{E\left(l_{i+1}(v)\right)}{L_{i}}+\frac{1}{\ln ^{3} \Delta}\right) X_{i} .
$$

It is simpler to show that $y_{i+1}^{\prime}$, rather than $y_{i+1}$, decreases quickly enough.

Lemma 5.3. For each vertex $v$ and $0 \leq i \leq\left\lceil\frac{10}{\alpha} \ln \Delta\right\rceil$, if $P(i)$ holds, then

$$
\operatorname{Pr}\left(y_{i+1}^{\prime}(v)>\left(\frac{E\left(l_{i+1}(v)\right)}{L_{i}}+\frac{1}{\ln ^{2} \Delta}\right) X_{i}\right)<\frac{1}{4} \Delta^{-\ln \Delta}
$$

Proof. Instead of showing that $y_{i+1}^{\prime}$ is concentrated directly, it is easier to look at the difference between $x_{i}^{\prime}$ and $y_{i+1}^{\prime}$. So we define

$$
R=x_{i}^{\prime}(v)-y_{i+1}^{\prime}(v)=\sum_{c \in L_{i}^{\prime}(v) \backslash L_{i+1}^{* \prime}(v)} t_{i}(v, c) .
$$

One trial can have the largest affect on $R$ by adding or removing a color $c$ from $L_{i}^{\prime}(v) \backslash L_{i+1}^{* \prime}(v)$. This changes $R$ by $t_{i}(v, c)$. Remember that in Modification 1, we discarded colors with large $t_{i}$ 's, so the value of $t_{i}(v, c)$ is at most $\Delta^{3 / 5}$.

Furthermore, for any $s>0$, we can certify that $X \geq s$, by showing at most $s \cdot \Delta^{-2 / 5}$ colors which are removed from $L_{i}^{\prime}(v)$ since $t_{i}(v, c) \geq \Delta^{2 / 5}$ for all $c \in L_{i}^{\prime}(v)$. We can certify that a color is removed from the list with just one trial: a neighbor of $v$ which is assigned that color. So Talagrand's Inequality with $c=\Delta^{3 / 5}, r=\Delta^{-2 / 5}$ and $t=\Delta^{19 / 20}$, yields:

$$
\begin{aligned}
\operatorname{Pr}\left(|R-E(R)|>\Delta^{19 / 20}\right) & \leq 4 e^{-\frac{\Delta^{19 / 10}}{32 \Delta^{6 / 5} \Delta^{-2 / 5}\left(\Delta+\Delta^{19 / 20}\right)}} \\
& \leq 4 e^{-\frac{1}{64} \Delta^{1 / 10}} \\
& <\frac{1}{4} e^{-\ln ^{2} \Delta}=\frac{1}{4} \Delta^{-\ln \Delta}
\end{aligned}
$$

By the linearity of expectation, Fact 2 and our bound on $E\left(y_{i+1}^{\prime}(v)\right)$, the above yields:

$$
\operatorname{Pr}\left(y_{i+1}^{\prime}(v)>\left(\frac{E\left(l_{i+1}(v)\right)}{L_{i}}+\frac{1}{\ln ^{2} \Delta}\right) x_{i}(v)\right)
$$




$$
\begin{aligned}
& \leq \operatorname{Pr}\left(y_{i+1}^{\prime}(v)>E\left(y_{i+1}^{\prime}(v)\right)+\Delta^{19 / 20}\right) \\
& \leq \operatorname{Pr}\left(|R-E(R)|>\Delta^{19 / 20}\right)<\frac{1}{4} \Delta^{-\ln \Delta}
\end{aligned}
$$

Now we prove that the parameter $t_{i}$ decreases quickly enough. We again use $L_{i}^{\prime}(v)$ to avoid small $t_{i}$ 's.

Lemma 5.4. For each vertex $v$, color $c \in L_{i}^{\prime}(v)$, and $0 \leq i \leq\left\lceil\frac{10}{\alpha} \ln \Delta\right\rceil$, if $P(i)$ holds, then

$$
\operatorname{Pr}\left(t_{i+1}(v, c)>\left(1-\frac{1}{\left(1+\frac{\alpha}{4}\right) \ln \Delta}\right) t_{i}(v, c)\right)<\frac{1}{2 \Delta} \Delta^{-\ln \Delta} .
$$

Proof. Instead of working with $t_{i+1}(v, c)$ directly, we work with $K$, the number of vertices in $N_{i}(v, c)$ which keep their color. Note that $t_{i+1}(v, c) \leq t_{i}(v, c)-K$.

We run into issues when $t_{i}(v, c)$ is at least $\sqrt{\Delta}$. So, we partition $N_{i}(v, c)$ into sets of size $o(\sqrt{\Delta})$. We know that $\left|N_{i}(v, c)\right|>\Delta^{2 / 5}$ since $c \in L_{i}^{\prime}(v)$, so we can partition $N_{i}(v, c)$ into $k$ nearly equal sized sets, $P_{1}, P_{2}, \ldots, P_{k}$, where $\Delta^{1 / 4} \leq\left|P_{j}\right| \leq 2 \Delta^{1 / 4}$ for all $1 \leq j \leq k$.

Let $K_{j}$ be the number of vertices in $P_{j}$ that keep their color. To prove $K_{j}$ is large enough, we define some new sets:

$$
\begin{aligned}
& A_{j}=\left\{v \in P_{j}: v \text { is activated }\right\} \\
& B_{j}=\left\{v \in A_{j}: v \text { is assigned a unique color among } A_{j}\right\} \\
& C_{j}=\left\{v \in B_{j}: v \text { keeps its color }\right\}
\end{aligned}
$$

Note that $K_{j} \geq\left|C_{j}\right|$.

We can analyze these random sets by carrying out the random choices in a specific sequence. First, we choose which members of $P_{j}$ are activated. This determines $A_{j}$. To get concentration for $\left|A_{j}\right|$, we can use the Chernoff Bound which yields:

$$
\begin{aligned}
\operatorname{Pr}\left(|| A_{j}\left|-\frac{\left|P_{j}\right|}{\ln \Delta}\right|>\frac{\left|P_{j}\right|}{\ln ^{2} \Delta}\right) & <2 e^{-\frac{\left|P_{j}\right|^{2}}{\ln ^{4} \Delta} \cdot \frac{\ln \Delta}{3\left|P_{j}\right|}} \\
& =e^{-\frac{\left|P_{j}\right|}{3 \ln ^{3} \Delta}} \leq e^{-\frac{\Delta^{1 / 4}}{3 \ln ^{3} \Delta}}<\frac{1}{6} \Delta^{-\ln \Delta-2}
\end{aligned}
$$

Next, we choose the colors assigned to $A_{j}$. Assuming ||$A_{j}\left|-\frac{\left|P_{j}\right|}{\ln \Delta}\right| \leq \frac{\left|P_{j}\right|}{\ln ^{2} \Delta}$, we bound the probability under this second set of random choices that $\left|B_{j}\right|$ is too small. If (at least) $\frac{\left|P_{j}\right|}{\ln ^{2} \Delta}$ vertices of $A_{j}$ are assigned the same color as another vertex in $A_{j}$, then there are at least $\frac{\left|P_{j}\right|}{3 \ln ^{2} \Delta}$ pairs of vertices $a, b \in A_{j}$, where $\mathrm{c}(a)=\mathrm{c}(b)$. The probability that there are at least $\frac{\left|P_{j}\right|}{3 \ln ^{2} \Delta}$ such pairs of vertices is at most:

$$
\text { (\# ways to choose } \left.\frac{\left|P_{j}\right|}{3 \ln ^{2} \Delta} \text { pairs from at most } \frac{\left|P_{j}\right|}{\ln \Delta}+\frac{\left|P_{j}\right|}{\ln ^{2} \Delta}\right)
$$




$$
\begin{aligned}
& \times \operatorname{Pr}(\text { every such pair }(a, b) \text { has } \mathrm{c}(a)=\mathrm{c}(b)) \\
& <\left(\left|P_{j}\right|\left(\frac{1}{\ln \Delta}+\frac{1}{\ln ^{2} \Delta}\right)\right)^{\frac{2\left|P_{j}\right|}{3 \ln ^{2} \Delta}} \cdot\left(\frac{1}{L_{i}}\right)^{\frac{\left|P_{j}\right|}{3 \ln ^{2} \Delta}} \\
& \leq\left(\frac{2\left|P_{j}\right|^{2}\left(\frac{1}{\ln \Delta}\right)^{2}}{L_{i}}\right)^{\frac{\left|P_{j}\right|}{3 \ln ^{2} \Delta}} \\
& \leq\left(\frac{8 \sqrt{\Delta}\left(\frac{1}{\ln \Delta}\right)^{2}}{\frac{\sqrt{\Delta}}{\ln \Delta}}\right)^{\frac{\left|P_{j}\right|}{3 \ln ^{2} \Delta}}[\text { by Fact } 1] \\
& \leq\left(\frac{1}{\ln ^{3} \Delta}\right)^{\frac{\Delta^{1 / 4}}{3 \ln ^{2} \Delta}} \leq \frac{1}{6} \Delta^{-\ln \Delta-2} \text { for } \Delta \text { sufficiently large }
\end{aligned}
$$

Therefore, if ||$A_{j}\left|-\frac{\left|P_{j}\right|}{\ln \Delta}\right| \leq \frac{\left|P_{j}\right|}{\ln ^{2} \Delta}$, then

$$
\begin{aligned}
\operatorname{Pr}\left(\left|B_{j}\right|<\left|P_{j}\right|\left(\frac{1}{\ln \Delta}-\frac{2}{\ln ^{2} \Delta}\right)\right) & \leq \operatorname{Pr}\left(\left|B_{j}\right|<\left|A_{j}\right|-\frac{\left|P_{j}\right|}{\ln ^{2} \Delta}\right) \\
& <\frac{1}{6} \Delta^{-\ln \Delta-2}
\end{aligned}
$$

Our final set of random choices is the activation and assignment choices for all vertices outside of $P_{j}$. Assuming that the outcomes of the other trials were such that $\left|B_{j}\right| \geq\left|P_{j}\right|\left(\frac{1}{\ln \Delta}-\frac{2}{\ln ^{2} \Delta}\right)$, we prove that $\left|C_{j}\right|$ is large enough with high probability under this final set of choices. Note that since $B_{j}$ contains only vertices which were assigned unique colors among $P_{j}$, only the colors assigned to vertices outside of $P_{j}$ can cause a vertex of $B_{j}$ to become uncolored. We start by computing the expected value of $\left|C_{j}\right|$. This computation is very similar to that in the proof of Lemma 5.1 and we can use the same argument as in Claim 5.1.1 to see that $E\left(\left|C_{j}\right|\right)$ is minimized over the rationals when all the $t_{i}$ 's are equal. Any vertex of $B_{j}-C_{j}$ must be uncolored because of a neighbor outside of $P_{j}$. Hence, $E\left(C_{j}\right)$ is bounded as follows.

$$
\begin{aligned}
E\left(\left|C_{j}\right|\right) & =\sum_{u \in B_{j}} \operatorname{Pr}(u \text { keeps its color }) \\
& \geq \sum_{u \in B_{j}} \sum_{\gamma \in L_{i}(u)} \frac{1}{L_{i}} \prod_{w \in N_{i}(u, \gamma)}\left(1-\frac{1}{\ln \Delta \cdot L_{i}}\right) \\
& =\sum_{u \in B_{j}} \sum_{\gamma \in L_{i}(u)} \frac{1}{L_{i}}\left(1-\frac{1}{\ln \Delta \cdot L_{i}}\right)^{t_{i}(u, \gamma)} \\
& \geq \sum_{u \in B_{j}} \sum_{\gamma \in L_{i}(u)} \frac{1}{L_{i}}\left(1-\frac{1}{\ln \Delta \cdot L_{i}}\right)^{\frac{X_{i}}{L_{i}}} \\
& =\left|B_{j}\right|\left(1-\frac{1}{\ln \Delta \cdot L_{i}}\right)^{\frac{x_{i}}{L_{i}}}
\end{aligned}
$$




$$
\begin{aligned}
& \geq\left|B_{j}\right|\left(1-\frac{X_{i}}{\ln \Delta \cdot L_{i}^{2}}\right) \text { for } \Delta \text { sufficiently large } \\
& \geq\left|B_{j}\right|\left(1-\frac{1}{\ln \Delta}\right)[\text { by Fact } 3]
\end{aligned}
$$

Instead of working with $C_{j}$ directly, we show concentration of the difference $R=\left|B_{j}\right|-\left|C_{j}\right|$. Note that changing the outcome of any one trial on a vertex not in $P_{j}$ can at most add or remove one vertex from $C_{j}$. Also, for any $s>0$, we can certify $R \geq s$, by finding $s$ vertices of $C_{j}$ that are uncolored and looking at the neighbor that got assigned the same color. Recalling that $\left|P_{j}\right| \geq \Delta^{1 / 4}$, and using the fact that $E(R) \leq\left|P_{j}\right|$, we can apply Talagrand's Inequality with $c=r=1$ and $t=\frac{\left|P_{j}\right|}{\ln ^{2} \Delta}$ to obtain:

$$
\begin{aligned}
\operatorname{Pr}\left(|R-E(R)|>\frac{\left|P_{j}\right|}{\ln ^{2} \Delta}\right) & <4 e^{-\frac{\left|P_{j}\right|^{2} / \ln ^{4} d}{32\left(\left|P_{j}\right|+\left|P_{j}\right|\right)}}=4 e^{-\frac{\left|P_{j}\right|}{64 \ln ^{4} \Delta}} \\
& \leq 4 e^{-\frac{\Delta^{1 / 4}}{64 \ln ^{4} \Delta}}<\frac{1}{6} \Delta^{-\ln \Delta-2}
\end{aligned}
$$

Now, using the linearity of expectation and our bound on $E\left(\left|C_{j}\right|\right)$, we get that if $\left|B_{j}\right| \geq\left|P_{j}\right|\left(\frac{1}{\ln \Delta}-\frac{2}{\ln ^{2} \Delta}\right)$, then

$$
\begin{aligned}
& \operatorname{Pr}\left(K_{j}<\left|P_{j}\right| \cdot \frac{1}{\left(1+\frac{\alpha}{4}\right) \ln \Delta}\right) \\
& \leq \operatorname{Pr}\left(\left|C_{j}\right|<\left|P_{j}\right|\left(\frac{1}{\ln \Delta}-\frac{2}{\ln ^{2} \Delta}\right)\left(1-\frac{1}{\ln \Delta}\right)-\frac{\left|P_{j}\right|}{\ln ^{2} \Delta}\right) \\
& \leq \operatorname{Pr}\left(\left|C_{j}\right|<\left|B_{j}\right|\left(1-\frac{1}{\ln \Delta}\right)-\frac{\left|P_{j}\right|}{\ln ^{2} \Delta}\right)<\frac{1}{6} \Delta^{-\ln \Delta-2}
\end{aligned}
$$

Since $K_{j} \geq\left|C_{j}\right|$, equations (2), (3) and (4) yield:

$$
\begin{aligned}
\operatorname{Pr}\left(K_{j}<\left|P_{j}\right| \cdot \frac{1}{\left(1+\frac{\alpha}{4}\right) \ln \Delta}\right) & <\frac{1}{6} \Delta^{-\ln \Delta-2}+\frac{1}{6} \Delta^{-\ln \Delta-2}+\frac{1}{6} \Delta^{-\ln \Delta-2} \\
& =\frac{1}{2} \Delta^{-\ln \Delta-2}
\end{aligned}
$$

Note that $K=\sum_{j=1}^{k} K_{j}, t_{i}(v, c)=\sum_{j=1}^{k}\left|P_{j}\right|$, and $k<\Delta$ to get the following:

$$
\operatorname{Pr}\left(K<t_{i}(v, c) \cdot \frac{1}{\left(1+\frac{\alpha}{4}\right) \ln \Delta}\right)<\Delta \cdot \frac{1}{2} \Delta^{-\ln \Delta-2}=\frac{1}{2 \Delta} \Delta^{-\ln \Delta}
$$

Since $t_{i+1}(v, c) \leq t_{i}(v, c)-K$, this completes our proof.

We are now ready to show that $x_{i}$ decreases quickly enough. We can use Lemmas 5.3 and 5.4 to prove Lemma 4.4. 
Proof of Lemma 4.4. As there are at most $\sqrt{(1+\alpha) \Delta}$ colors in $L_{i+1}^{\prime}$, Lemma 5.4 implies that with probability at least

$$
1-\sqrt{(1+\alpha) \Delta} \cdot \frac{1}{2 \Delta} \Delta^{-\ln \Delta} \geq 1-\frac{1}{2} \Delta^{-\ln \Delta}
$$

for every $c \in L_{i+1}^{\prime}$ we have:

$$
t_{i+1}(v, c) \leq\left(1-\frac{1}{\left(1+\frac{\alpha}{4}\right) \ln \Delta}\right) t_{i}(v, c)
$$

By Lemma 5.3, with probability at least $1-\frac{1}{2} \Delta^{-\ln \Delta}$, we have:

$$
y_{i+1}^{\prime}(v) \leq\left(\frac{E\left(l_{i+1}(v)\right)}{L_{i}}+\frac{1}{\ln ^{2} \Delta}\right) X_{i}
$$

From the proof of Lemma 4.3, we know that the following holds with probability at least $\frac{1}{4} \Delta^{-\ln \Delta}$ :

$$
l_{i+1}(v) \geq E\left(l_{i+1}(v)\right)-\frac{L_{i}}{\ln ^{2} \Delta}
$$

Let $x_{i}^{*}$ be the following new parameter.

$$
x_{i}^{*}(v)=\sum_{c \in L_{i}^{*}(v)} t_{i}(v, c)
$$

This is the same as $x_{i}$, except the value is measured before the truncation step. If (5), (6) and (7) hold, then:

$$
\begin{aligned}
x_{i+1}^{*}(v) & =\sum_{c \in L_{i+1}^{* \prime}(v)} t_{i+1}(v, c)+\sum_{c \in L_{i+1}^{*}(v) \backslash L_{i+1}^{* \prime}(v)} t_{i+1}(v, c) \\
& \left.\leq\left(\sum_{c \in L_{i+1}^{* \prime}(v)} t_{i+1}(v, c)\right)+\sqrt{(1+\alpha) \Delta} \cdot \Delta^{2 / 5} \text { [by definition of } L_{i}^{* \prime}(v)\right] \\
& \left.\leq\left[\sum_{c \in L_{i+1}^{* \prime}(v)}\left(1-\frac{1}{\left(1+\frac{\alpha}{4}\right) \ln \Delta}\right) t_{i}(v, c)\right]+\frac{X_{i}}{\ln ^{2} \Delta} \text { [by (5) and Fact } 2\right] \\
& \leq\left(1-\frac{1}{\left(1+\frac{\alpha}{4}\right) \ln \Delta}\right)\left(\frac{E\left(l_{i+1}(v)\right)}{L_{i}}+\frac{1}{\ln ^{2} \Delta}\right) X_{i}+\frac{X_{i}}{\ln ^{2} \Delta}[\text { by }(6)] \\
& \leq\left[\left(1-\frac{1}{\left(1+\frac{\alpha}{4}\right) \ln \Delta}\right)\left(\frac{E\left(l_{i+1}(v)\right)}{L_{i}}\right)+\frac{2}{\ln ^{2} \Delta}\right] X_{i} \\
& \leq\left[\left(1-\frac{1}{\left(1+\frac{\alpha}{4}\right) \ln \Delta}\right)\left(\frac{l_{i+1}(v)+\frac{L_{i}}{\ln ^{2} \Delta}}{L_{i}}\right)+\frac{2}{\ln ^{2} \Delta}\right] X_{i}[\text { by }(7)] \\
& \leq\left[\left(1-\frac{1}{\left(1+\frac{\alpha}{4}\right) \ln \Delta}\right)\left(\frac{l_{i+1}(v)}{L_{i}}\right)+\frac{3}{\ln ^{2} \Delta}\right] X_{i}
\end{aligned}
$$


To bound $x_{i+1}$, we recall that in Step 5 , we are removing the $c$ 's with the largest $t_{i}$ 's. We will keep a proportion of $\frac{L_{i+1}}{l_{i+1}(v)}$ of the colors in $v$ 's list, so, by our choice of which $c$ 's we remove, we get the following:

$$
\begin{aligned}
x_{i+1}(v) & \leq \frac{L_{i+1}}{l_{i+1}(v)} \cdot x_{i+1}^{*}(v) \\
& \leq \frac{L_{i+1}}{l_{i+1}(v)} \cdot\left[\left(1-\frac{1}{\left(1+\frac{\alpha}{4}\right) \ln \Delta}\right)\left(\frac{l_{i+1}(v)}{L_{i}}\right)+\frac{3}{\ln ^{2} \Delta}\right] X_{i} \\
& \leq\left[\left(1-\frac{1}{\left(1+\frac{\alpha}{4}\right) \ln \Delta}\right)\left(\frac{L_{i+1}}{L_{i}}\right)+\frac{3}{\ln ^{2} \Delta}\right] X_{i} \\
& \leq\left[\left(1-\frac{1}{\left(1+\frac{\alpha}{4}\right) \ln \Delta}\right)\left(1-\frac{1}{\left(1+\frac{3 \alpha}{4}\right) \ln \Delta}\right)+\frac{3}{\ln ^{2} \Delta}\right] X_{i} \\
& \leq\left(1-\frac{2}{\left(1+\frac{\alpha}{2}\right) \ln \Delta}\right) X_{i} \quad \text { for } \Delta \text { sufficiently large } \\
& \leq\left(1-\frac{1}{\left(1+\frac{\alpha}{2}\right) \ln \Delta}\right)^{2} X_{i}=X_{i+1}
\end{aligned}
$$

This holds as long as (5), (6) and (7) hold, which occurs with probability at least $\frac{1}{4} \Delta^{-\ln \Delta}+\frac{1}{2} \Delta^{-\ln \Delta}+\frac{1}{4} \Delta^{-\ln \Delta}=\Delta^{-\ln \Delta}$.

\section{Concluding Remarks}

While our bound is asymptotically optimal, there are still ways in which it could be improved. As mentioned above, there are examples where $\chi_{a}(G)=\sqrt{\Delta}+1$ (e.g. $K_{n}^{2}$ for certain $n$ ). In response to Reed's paper [21], Bohman and Holzman [3] constructed a graph $G$ with lists $L(v)$ for every vertex $v$ such that: (i) for each vertex $v$ and color $c \in L(v)$, the number of neighbors $u$ of $v$ with $c \in L(u)$ is at most $d$, (ii) for each vertex $v,|L(v)|=d+1$, and (iii) $G$ is uncolorable from these lists. If we convert this into an example for adaptable coloring, as described in Section 1, we get an uncolorable graph $G$ with maximum degree $\Delta=d(d+1)$ and lists of size $d+1$; thus $\operatorname{ch}_{a}(G) \geq \sqrt{\Delta}+2$.

Question 1. Is there a constant $k$ such that $\operatorname{ch}_{a}(G) \leq \sqrt{\Delta}+k$ for every graph $G$ ?

Determining whether $\chi_{a}$ grows with $\chi$ may be the most important question in this area. We mentioned above that $\chi_{a}$ has a lower bound of order $\chi / \sqrt{n \ln \chi}$ [12]. We would like to know whether there is a bound that is only in terms of $\chi$.

Question 2. Is there a function $f$ tending to infinity such that $\chi_{a}(G) \geq$ $f(\chi(G))$ for every graph $G$ ? 
Conceivably, $f$ could be as high as $\Theta(\sqrt{\chi})$. The complete graph has $\chi_{a}=$ $\Theta(\sqrt{\chi})[6]$, but no example has been found with a smaller adaptable chromatic number in terms of $\chi$.

Question 3. Are there any graphs $G$ with $\chi_{a}(G)=o(\sqrt{\chi(G)})$ ?

Intuitively, $\chi_{a}$ is typically much less than $\chi$. There are constructions, however, that demonstrate that $\chi_{a}$ can be equal to $\chi[14,15]$. These constructions rely on blowing up graphs in a way that maintains $\chi$ while increasing $\chi_{a}$; they have at least $\chi^{2}$ vertices but contain the $\chi$-clique. Thus these graphs are far from critical. It would be interesting to see how close $\chi_{a}$ can get to $\chi$ for critical graphs. It is easily seen that a critical graph cannot have $\chi_{a}=\chi$ (for example this follows from Proposition 2.1 in [15]), but nothing more is known.

Question 4. Are there any critical graphs $G$ with $\chi_{a}(G)=\chi(G)-1$ ?

The largest we can get $\chi_{a}$ to be in terms of $\chi$ for critical graphs may in fact be much worse than this.

Question 5. Are there any critical graphs $G$ with $\chi_{a}(G)=\omega(\sqrt{\chi(G)})$ ?

\section{References}

[1] N. Alon and J. Spencer, The probabilistic method, 2nd ed., Wiley, New York, 2000.

[2] A. Archer, On the upper chromatic numbers of the reals, Discrete Math, 214:65-75, 2000.

[3] T. Bohman and R. Holzman, On a list coloring conjecture of Reed, J. Graph Theory, 41:106-109, 2002.

[4] K. Chandrasekaran, N. Goyal and B. Haeupler, Deterministic algorithms for the Lovász Local Lemma, to appear in Proc. of ACM-SIAM Symposium on Discrete Algorithms (SODA), 2010.

[5] H. Chernoff, A measure of the asymptotic efficiency for tests of a hypothesis based on the sum of observations, Annuals of Mathematical Statistics, 23:493-509, 1952.

[6] P. Erdős and A. Gyárfás, Split and balanced colorings of complete graphs, Discrete Math, 200 (1999), 79-86.

[7] P. Erdős and L. Lovász, Problems and results on 3-chromatic hypergraphs and some related questions, in: Infinite and Finite Sets (A. Hajnal et al., eds.), Colloq. Math. Soc. J. Bolyai 11, North Holland, Amsterdam, The Netherlands (1975), 609-627.

[8] J. Esperet, M. Montassier and X. Zhu, Adapted list colouring of planar graphs, manuscript, 2007. 
[9] T. Feder and P. Hell, Full constraint satisfaction problems, SIAM J. Computing, 36 (2006), 230-246.

[10] T. Feder, P. Hell, S. Klein and R. Motwani, Complexity of list partitions, SIAM J. Discrete Mathematics, 16 (2003), 449-478.

[11] T. Feder, P. Hell and W. Xie, Matrix partitions with finitely many obstructions, Electronic Notes in Discrete Math, 28 (2007), 371-378.

[12] J.E. Greene, Chromatic capacities of graphs and hypergraphs, Discrete Math 281 (2004), 197-207.

[13] P. Haxell, A note on vertex list colouring, Combinatorics, Probability and Computing, 10 (2001), 345-347.

[14] P. Hell, Z. Pan, T. Wong and X. Zhu, Adaptable colouring of graph products, preprint.

[15] P. Hell and X. Zhu, Adaptable chromatic number of graphs, European J. Combinatorics, 29(4) (2008), 912-921.

[16] A. Kostochka and X. Zhu, Adapted list coloring of graphs and hypergraphs, SIAM J. Discrete Math, to appear.

[17] M. Molloy and B. Reed, Colouring graphs when the number of colours is nearly the maximum degree, preprint.

[18] M. Molloy and B. Reed, Graph colouring and the probabilistic method, Springer-Verlag, Berlin, 2001.

[19] R. Moser. A constructive proof of the general Lovász Local Lemma, in Proc. of the 41st Annual ACM Symposium on Theory of Computing (STOC) (2009), 343-350.

[20] R. Moser and G. Tardos, A constructive proof of the general Lovász Local Lemma, preprint.

[21] B. Reed, The list colouring constants, J. Graph Theory, 31(2) (1999) 149153.

[22] B. Reed and B. Sudakov, Asymptotically the list colouring constants are 1, J. Combinatorial Theory Ser. B, 86(1) (2002), 27-37.

[23] M. Talagrand, Concentration of measure and isoperimetric inequalities in product spaces, Publications Mathématiques de l'IHÉS, 81 (1995), 73-205. 